\title{
Patterns of endocrine reactivity in patients with recent myocardial infarction Clinical and biochemical correlations: trial of endocrine therapy
}

\author{
L. Ceremużyński, J. Kuch, L. Markiewicz ${ }^{1}$, J. Lawecki, and J. Taton ${ }^{2}$ \\ From the Institute of Cardiology of the Medical Academy, Warsaw, Lindley'a Str. 4, Poland
}

The following endocrine function parameters were studied serially in a group of Io patients with recent myocardial infarction: blood and urinary levels of epinephrine and norepinephrine, urinary excretion of vanillyl-mandelic acid; protein-bound iodine, Hamolsky test (Hamolsky, Stein, and Freedberg, 1957); blood insulin; 24-hour urinary excretion of I7-hydroxycorticoids, sodium, and potassium.

The acute phase of myocardial infarction, especially in those patients with a severe clinical course (rhythm disturbances, coronary insufficiency, circulatory failure), was associated with disturbed endocrine reactivity.

The most frequent and the earliest feature was the increased level of the 24-hour urinary excretion of epinephrine, combined with a pronounced decrease in blood insulin level.

Later in the course of the disease, as the adrenergic reactivity returned to normal, there was an increase in blood insulin to normal levels.

In 3 patients with severe clinical symptoms of acute myocardial infarction, there were, in addition to the increased 24-hour urinary excretion of catecholamines, a decreased blood insulin, higher than normal levels of protein-bound iodine, and of the Hamolsky test. One of these patients developed hypoadrenia.

It is possible that the abnormal endocrine reactions accelerate the catabolic processes within cardiac tissue (catecholamines, thyroid hormones), especially when there is a possible functional deficiency of hormones, occurring as a general adaptation reaction to stress (cortisol, insulin). The disturbances that follow may be dangerous for the patient.

Acute myocardial infarction could be considered as a pathological stimulus inducing a state of stress with general reflex responses. The endocrine reactions to this stress include enhanced secretion of catecholamines (Aleksandrow, Januszewicz, and Wocial, I966; Ceremużyński, I970; Gazes, Richardson, and Woods, 1959; Kuschke and Schneider, 1960), glycocorticoids (Ceremużyński, 1967a; Klein and Palmer, 1963; Logan and Murdoch, I966), aldosterone (Arora, I965; Ceremużyński, 1970; Wolff et al., 1956), modified

Received 15 October 1969.

1 Present address: Central Research Institute for Labour Protection.

2 Present address: Third Department of Internal Medicine, Medical Academy, Warsaw. activity of the thyroid gland, as well as some secondary alterations in metabolism (Ceremużyński, 1970).

The patterns of hormonal reactions to acute myocardial infarction are intense in some patients. The clinical course of the infarction in such patients is usually complicated or fatal (Ceremużyński, 1967a; Klein and Palmer, 1963).

Valori, Thomas, and Shillingford (1967), as well as Januszewicz et al. (1968), proved that cardiac rhythm disturbances appeared more frequently in patients with recent myocardial infarction who had enhanced excretion of catecholamines. The determination of catecholamines in blood and urine in 30 patients with myocardial infarction showed that the differences between the increased levels of 
catecholamines in infarction with a complicated clinical course (intractable coronary chest pain, rhythm disturbances) and the normal secretion in mild forms of infarction appeared during the first week of the disease, and that the main difference was in the urinary excretion of epinephrine (Ceremużyński, in press).

Many investigators found a considerably increased secretion of cortisol during a severe infarction (especially in patients with shock), in the main during the acute early phase. As has been recently shown, it is very often followed by a decrease in the excretion of urinary catabolites of glycocorticoids, and can be as low as the levels occurring in hypoadrenia (perhaps as a result of the exhaustion of the suprarenal cortex ?). This phenomenon often occurs in such patients 2 to 3 weeks after the infarction. These symptoms are accompanied by some pathological manifestations in the circulatory system (cardiac rhythm disturbances, coronary chest pain, cardiac failure) (Ceremużyński, 1967a).

It has recently been stated as a result of the examination of 50 patients with myocardial infarction that some parameters of thyroid activity (protein-bound iodine, Hamolsky test) in serial determinations show release of thyroid hormones during the first few days after the infarction. In some patients with an unfavourable clinical course, increased levels on the thyroid tests have been found also in the fourth week of the disease (Ceremużynski, in press).

In addition to adrenal and thyroid gland secretory disturbances, in many patients during the acute phase of a severe infarction there is a lower level of immunoradioactive insulin as well as an increased excretion of aldosterone and a decrease in the excretion of sodium (Ceremużyński, in press). The endocrine abnormalities sometimes quantitatively correspond to the values found, e.g. in patients with the thyro-cardiac syndrome, phaeochromocytoma, hypoadrenia, or diabetes mellitus.

It seems important to extend the complex clinical parameters monitored in recent myocardial infarction and add the observations on endocrine functional activity. Such a procedure may enable remedial treatment when disturbed hormonal homeostasis occurs, as, for example, when abnormal values of $p \mathrm{H}$ or electrolyte disturbances are diagnosed.

This report presents observations concerning the group of patients suffering from recent myocardial infarction in whom some biochemical parameters of endocrine reactivity have been serially determined. Compensa- tory therapy has been administered to some of these patients.

\section{Subjects and methods}

Hormonal studies were performed in ro patients. The course of myocardial infarction was severe in 5 of them, and in the other 5 there were no unfavourable clinical symptoms. All the patients were men aged from 4I to 65, who were in hospital in the Institute of Cardiology of the Warsaw Medical School (first in the intensive care unit and then in the regular hospital ward) because of recent myocardial infarction. The diagnosis of myocardial infarction was based on the clinical picture, electrocardiogram, and enzymatic tests.

The following hormonal studies were performed simultaneously: epinephrine and norepinephrine in blood and in 24-hour urine, using the fluorometric method, according to Bertler, Carlsson, and Rosengren (1958); vanillyl mandelic acid in 24-hour urine according to Woiwod and Knight (I96r); protein-bound iodine according to Barker (Barker and Klitgaard, 1952) and the Hamolsky test (Hamolsky et al., I957). The determination of I7-OHCS was carried out according to the SilberPorter method (I954). Immunoradioactive insulin in blood was determined with Melani technique (Melani et al., 1965). The daily excretion of $\mathrm{Na}$ and $\mathrm{K}$ ions in urine was estimated as well.

These examinations were performed on the second, fourth, and sixth days after the onset of myocardial infarction and also in the second, third, and fourth week (on the third or fourth day of each week). Since, in some cases, it was necessary to administer drugs that might have affected the endocrine function substantially, the time schedule of some determinations was not always maintained.

\section{Patients with clinically unfavourable infarc-} tion (Table I)

Case I A man of 60 , with coronary chest pain for 4 years, had his first myocardial infarction two years earlier. A year previously he had four episodes of acute coronary insufficiency without recent myocardial necrosis. $\mathrm{He}$ was admitted to hospital because of the recent myocardial infarction (his second) with severe pulmonary oedema. On the fourth day multiple ventricular ectopic beats were monitored, and these were followed by ventricular fibrillation which was treated with external electrical countershock.

A few hours before ventricular fibrillation occurred pathologically high levels of epinephrine and a low level of immunoradioactive insulin (blood sugar $143 \mathrm{mg}$./100 ml.) were found in blood samples, as well as an increased urinary excretion of epinephrine. The last determination of $17-\mathrm{OHCS}$ before ventricular fibrillation occurred showed a low level (3.2 mg. daily). At the same time the excretion of urinary sodium was much decreased, reaching a lower level than that of potassium. Therefore, in addition to routine treatment with digoxin, frusemide, procainamide, prenylamine, and pentaerythrityl tetranitrate, 
TABLE I Hormonal reactivity in patients with severe myocardial infarction

\begin{tabular}{|c|c|c|c|c|c|c|c|c|c|c|c|c|c|}
\hline & & \multicolumn{11}{|c|}{ Investigations } & \multirow[b]{3}{*}{ Clinical symptoms } \\
\hline & & \multicolumn{5}{|l|}{ Blood } & \multicolumn{6}{|l|}{ Urine } & \\
\hline & & $\begin{array}{l}\text { Protein- } \\
\text { bound } \\
\text { iodine }\end{array}$ & $\begin{array}{l}\text { Hamol- } \\
\text { sky } \\
\text { test }\end{array}$ & $\begin{array}{l}\text { Immuno- } \\
\text { radio- } \\
\text { active } \\
\text { insulin }\end{array}$ & $\begin{array}{l}\text { Adrena- } \\
\text { line }\end{array}$ & $\begin{array}{l}\text { Nor- } \\
\text { adrena- } \\
\text { line }\end{array}$ & $\begin{array}{l}\text { Adrena- } \\
\text { line }\end{array}$ & $\begin{array}{l}\text { Nor- } \\
\text { adrena- } \\
\text { line }\end{array}$ & $\begin{array}{l}\text { Vanillyl } \\
\text { mandelic } \\
\text { acid }\end{array}$ & $\begin{array}{l}\text { 17- } \\
\mathrm{OHCS}\end{array}$ & $K+$ & $\mathrm{Na}+$ & \\
\hline \multirow{3}{*}{$\begin{array}{l}\text { Case I } \\
\text { Days }\end{array}$} & & & & & & & & & & & & & \\
\hline & 2 & $5 \cdot 2$ & $17 \cdot 0$ & - & $c \cdot 5$ & $7 \cdot 3$ & $8 \cdot 5$ & $14 \cdot 8$ & - & $3 \cdot 2$ & I. 7 & I. 7 & $\begin{array}{l}\text { Cardiac pain, cardiac } \\
\text { rhythm disturbances, } \\
\text { circulatory insuffici- } \\
\text { ency }\end{array}$ \\
\hline & 4 & $4 \cdot 0$ & $15 \cdot 5$ & $14 \cdot 0$ & $3 \cdot 8$ & $1 \cdot 0$ & $13 \cdot 6$ & $7 \cdot 8$ & $7 \cdot 0$ & $13 \cdot 8$ & $2 \cdot 5$ & 0.9 & $\begin{array}{l}\text { Cardiac pain, cardiac } \\
\text { rhythm disturbances, } \\
\text { circulatory insuffici- } \\
\text { ency }\end{array}$ \\
\hline & 6 & - & 18.5 & $14 \cdot 0$ & $2 \cdot 7$ & $I \cdot 0$ & $4 \cdot 8$ & $27 \cdot 0$ & $9 \cdot 1$ & $4 \cdot 2$ & $1 \cdot 2$ & 0.7 & Circulatory insufficiency \\
\hline \multirow[t]{3}{*}{ Weeks } & 2 & $6 \cdot 3$ & $14 \cdot 5$ & - & 2.5 & 0.3 & $2 \cdot 4$ & $14 \cdot 9$ & $9 \cdot 3$ & $5 \cdot 2$ & $2 \cdot 8$ & $3 \cdot 6$ & Circulatory insufficiency \\
\hline & 3 & 3.8 & $15 \cdot 5$ & - & I. 4 & $4 \cdot 6$ & $4 \cdot I$ & $47 \cdot I$ & 6.8 & 54 & $2 \cdot 9$ & $2 \cdot 6$ & Circulatory insufficiency \\
\hline & 4 & $6 \cdot 1$ & $16 \cdot 0$ & $8 \cdot 0$ & $2 \cdot 2$ & 140 & $9 \cdot 1$ & $35 \cdot 4$ & $9 \cdot 6$ & $7 \cdot 8$ & - & - & Circulatory insufficiency \\
\hline \multirow{4}{*}{$\begin{array}{l}\text { Case } 2 \\
\text { Days }\end{array}$} & & & & & & & & & & & & & \\
\hline & 2 & 3.5 & - & $14 \cdot 0$ & $1 \cdot 2$ & I. 3 & $3 \cdot 6$ & 85.5 & $6 \cdot 4$ & - & $I \cdot 0$ & 0.2 & $\begin{array}{l}\text { Cardiac pain, circulatory } \\
\text { insufficiency }\end{array}$ \\
\hline & 4 & - & - & - & - & - & - & - & - & - & - & - & $\begin{array}{l}\text { Cardiac pain, circulatory } \\
\text { insufficiency }\end{array}$ \\
\hline & 6 & 5.5 & 15.5 & $22 \cdot 0$ & I 2 & $2 \cdot 0$ & 19.9 & $36 \cdot 9$ & 10.9 & $5 \cdot 2$ & 2.5 & $2 \cdot I$ & $\begin{array}{l}\text { Cardiac pain, circulatory } \\
\text { insufficiency }\end{array}$ \\
\hline \multirow{2}{*}{\multicolumn{2}{|c|}{$\begin{array}{l}\text { Weeks } 2 \\
\text { Death }\end{array}$}} & $5 \cdot 6$ & 18.5 & - & $1 \cdot 3$ & I.7 & $28 \cdot 0$ & 10.9 & $3 \cdot 4$ & 20 & I. 4 & 0.6 & $\begin{array}{l}\text { Cardiac pain, circulatory } \\
\text { insufficiency }\end{array}$ \\
\hline & & & & & & & & & & & & & \\
\hline \multirow{4}{*}{$\begin{array}{l}\text { Case } 3 \\
\text { Days }\end{array}$} & & & & & & & & & & & & & \\
\hline & 2 & $7 \cdot 2$ & $9 \cdot 0$ & 8.0 & 0.3 & $7 \cdot 4$ & - & - & - & - & $I \cdot 2$ & 0.6 & $\begin{array}{l}\text { Cardiac pain, circulatory } \\
\text { insufficiency, cardiac } \\
\text { rhythm disturbances }\end{array}$ \\
\hline & 4 & $4 \cdot 0$ & $22 \cdot 0$ & - & 0.5 & $4 \cdot 3$ & $9 \cdot 0$ & 65.0 & 5.5 & $2 \cdot 8$ & 0.8 & $1 \cdot 0$ & $\begin{array}{l}\text { Cardiac pain, circulatory } \\
\text { insufficiency, cardiac } \\
\text { rhythm disturbances }\end{array}$ \\
\hline & 6 & - & II. 5 & - & $2 \cdot 0$ & $I \cdot I$ & - & - & IO. I & - & $1 \cdot 3$ & 0.7 & $\begin{array}{l}\text { Cardiac pain, circulatory } \\
\text { insufficiency, cardiac } \\
\text { rhythm disturbances }\end{array}$ \\
\hline \multirow[t]{2}{*}{ Weeks } & & 5.8 & 14.5 & $34 \cdot 0$ & 0.2 & 10.4 & $9 \cdot 7$ & 184.0 & I 8.9 & $7 \cdot 4$ & $2 \cdot 9$ & $1 \cdot 7$ & $\begin{array}{l}\text { Cardiac pain, circulatory } \\
\text { insufficiency }\end{array}$ \\
\hline & 3 & 50 & 12.5 & - & 0.5 & $2 \cdot 6$ & - & $30 \cdot 2$ & 10.3 & $6 \cdot 5$ & - & - & - \\
\hline \multirow{4}{*}{$\begin{array}{l}\text { Case } 4 \\
\text { Days }\end{array}$} & & & & & & & & & & & & & \\
\hline & 2 & $7 \cdot 3$ & $19 \cdot 0$ & $\circ$ & 0.7 & 6.7 & 17 & IIII & 3.I & $5 \cdot 0$ & $2 \cdot 2$ & I.O & $\begin{array}{l}\text { Cardiac pain, circulatory } \\
\text { insufficiency, cardiac } \\
\text { rhythm disturbances }\end{array}$ \\
\hline & 4 & - & - & - & - & - & - & - & - & - & - & - & $\begin{array}{l}\text { Cardiac pain, circulatory } \\
\text { insufficiency, cardiac } \\
\text { rhythm disturbances }\end{array}$ \\
\hline & 6 & - & 17.5 & - & 4.0 & 4.4 & $9 \cdot 7$ & 119.3 & $4 \cdot 3$ & 5.8 & I. 4 & 0.8 & $\begin{array}{l}\text { Circulatory insufficien- } \\
\text { cy, cardiac rhythm } \\
\text { disturbances }\end{array}$ \\
\hline \multirow[t]{2}{*}{ Weeks } & 2 & $\begin{array}{r}6 \cdot 3 \\
-\end{array}$ & $\begin{array}{l}17 \cdot 0 \\
13 \cdot 0\end{array}$ & $\bar{z}$ & 3.5 & 10.9 & 3.7 & $50 \cdot 0$ & $4 \cdot 9$ & 5.7 & $1 \cdot 8$ & I.8 & Circulatory insufficiency \\
\hline & $\begin{array}{l}3 \\
4\end{array}$ & $\overline{5.5}$ & $\begin{array}{l}13 \cdot 0 \\
23 \cdot 0\end{array}$ & $\overline{14} \cdot 0$ & $\begin{array}{l}0.6 \\
3.0\end{array}$ & $\begin{array}{l}7.6 \\
1.8\end{array}$ & $\begin{array}{l}6 \cdot 5 \\
4 \cdot 7\end{array}$ & $\begin{array}{l}40.5 \\
53.6\end{array}$ & $\begin{array}{l}5.5 \\
4 \cdot 7\end{array}$ & $\begin{array}{l}7 \cdot 4 \\
5 \cdot 4\end{array}$ & $\begin{array}{l}4 \cdot 3 \\
1 \cdot 0\end{array}$ & $2 \cdot 3$ & $\bar{z}$ \\
\hline \multirow{3}{*}{$\begin{array}{l}\text { Case } 5 \\
\text { Days }\end{array}$} & & & & & & & & & & & & 13 & - \\
\hline & 2 & - & - & $2 \cdot 0$ & 0.7 & 3.8 & $28 \cdot I$ & $60 \cdot 0$ & $4 \cdot 6$ & $4 \cdot 6$ & $\mathbf{I} \cdot \mathbf{I}$ & 0.5 & $\begin{array}{l}\text { Cardiac pain, circulatory } \\
\text { insufficiency, cardiac } \\
\text { rhythm disturbances }\end{array}$ \\
\hline & 4 & II $\cdot 8$ & $26 \cdot 0$ & - & $7 \cdot 3$ & $7 \cdot 8$ & II $\cdot 3$ & $132 \cdot 6$ & $8 \cdot 4$ & $10 \cdot 7$ & $2 \cdot 2$ & $3 \cdot 0$ & $\begin{array}{l}\text { Cardiac pain, circulatory } \\
\text { insufficiency, cardiac } \\
\text { rhythm disturbances }\end{array}$ \\
\hline \multirow{4}{*}{ Weeks } & & $4 \cdot 8$ & - & $12 \cdot 0$ & $\mathbf{I} \cdot \mathbf{O}$ & 30 & $15 \cdot 8$ & $55 \cdot 2$ & - & $7 \cdot 7$ & $2 \cdot \mathbf{I}$ & $5 \cdot 3$ & Circulatory insufficiency \\
\hline & 2 & 47 & $18 \cdot 0$ & $12 \cdot 0$ & $2 \cdot I$ & $5 \cdot 6$ & 9.5 & $34 \cdot 8$ & - & $6 \cdot 2$ & $1 \cdot 4$ & $\mathbf{2 \cdot 2}$ & Circulatory insufficiency \\
\hline & 3 & 5.8 & $21 \cdot 5$ & $16 \cdot 0$ & 0.6 & $8 \cdot 3$ & - & - & $6 \cdot 4$ & $2 \cdot 9$ & $1 \cdot 2$ & $\mathbf{I} \cdot 9$ & Circulatory insufficiency \\
\hline & 4 & $5 \cdot 7$ & $16 \cdot 5$ & $10 \cdot 0$ & 2.I & $2 \cdot 3$ & $2 \cdot 8$ & $16 \cdot 9$ & $4 \cdot 2$ & $2 \cdot 5$ & $\mathbf{I} \cdot \mathbf{I}$ & $2 \cdot 3$ & - \\
\hline \multicolumn{2}{|l|}{$\begin{array}{l}\text { Normal } \\
\text { ranges }\end{array}$} & $\begin{array}{l}5 \cdot 3 \pm \mathrm{I} \cdot 7 \\
\mu \mathrm{g} . / 100 \\
\mathrm{ml} .\end{array}$ & $\begin{array}{c}15 \cdot 0 \pm 2 \cdot 5 \\
\%\end{array}$ & $\begin{array}{c}20-30 \\
\mu U^{2}\end{array}$ & $\begin{array}{c}0.6 \pm 0.5 \\
\mu \mathrm{g} . / 1 .\end{array}$ & $\begin{array}{c}2 \cdot 5 \pm \mathrm{I} \cdot \mathrm{I} \\
\mu \mathrm{g} . / 1 .\end{array}$ & $\begin{array}{c}3 \cdot 5 \pm \mathrm{r} \cdot 3 \\
\mu \mathrm{g.} / 24 \\
\mathrm{hr} .\end{array}$ & $\begin{array}{l}26 \cdot 9 \pm 7 \cdot 7 \\
\mu \mathrm{g} . / 24 \\
\mathrm{hr} .\end{array}$ & $\begin{array}{l}4 \cdot 6 \pm \mathrm{I} \cdot 2 \\
\mathrm{mg} \cdot / 24 \\
\mathrm{hr} .\end{array}$ & $\begin{array}{l}5 \cdot 0 \pm \mathrm{r} \cdot 5 \\
\mathrm{mg} \cdot / 24 \\
\mathrm{hr} .\end{array}$ & $\begin{array}{l}2 \cdot 0-3 \cdot 0 \\
\text { g. } / 24 \mathrm{hr} .\end{array}$ & $\begin{array}{l}3.0-5 \\
\text { g. } / 24 \mathrm{~h}\end{array}$ & \\
\hline
\end{tabular}


which had given little clinical benefit, cortisone acetate, $50 \mathrm{mg}$. daily, and later $25 \mathrm{mg}$. daily, was administered as well as crystalline insulin, 6 units twice daily. While on this therapy the patient's general state slowly improved. He was discharged after 8 weeks of treatment.

Case 2 A man of 63, with recent myocardial infarction with pulmonary oedema. During the first week in hospital coronary chest pain recurred, as well as the symptoms of cardiac asthma. In spite of the usual treatment with deslanoside, frusemide, and pentaerythrityl tetranitrate, the patient's condition deteriorated during the second week. Attacks of pulmonary oedema occurred, and he died in acute circulatory failure. One week before his death very high levels of adrenaline in 24hour urine samples had been found. These levels increased still more two days before death. A considerable fall in the urinary excretion of 17OHCS also occurred at the same time. Hydrocortisone hemisuccinate, IoO mg. daily, was then added to the routine drugs. The excretion of sodium in the urine was considerably reduced during the whole course of his illness and was lower than the potassium excretion.

- Case 3 A man of 57 had arterial hypertension and coronary insufficiency for ro years. His first myocardial infarction had occurred Io months previously. He was admitted to hospital because of a second recent myocardial infarction. On admission his general condition was serious. During the first few days in hospital there were signs of cardiac failure (dyspnoea, râles), coronary chest pains, and rhythm disorders (multifocal and multiple ventricular ectopic beats). Hormonal determinations indicated an increase of thyroid activity, a low level of immunoradioactive insulin (blood sugar at this time was $194 \mathrm{mg}$./ $100 \mathrm{ml}$.), and low levels of urinary $17-\mathrm{OHCS}$, as in hypoadrenia. Urinary excretion of sodium was very low, again lower than the excretion of potassium. The patient was treated initially with dolantinum, prenylamine, dipyridamole, deslanoside, and quinidine. After four days, potassium chloride and cortisone acetate ( $25 \mathrm{mg}$. daily) as well as crystalline insulin (6 units twice daily) were administered. The patient's general condition improved in the course of the following few days. There was a parallel return to normal endocrine reactions. He was discharged after 7 weeks of treatment.

Case 4 A man of 44, in hospital because of a recent myocardial infarction which occurred two months after the first one. His general condition was serious, with signs of cardiac failure (dyspnoea, cyanosis, râles, gallop rhythm). The hormonal determinations performed on the second day showed increased thyroid activity, low levels of immunoradioactive insulin (blood sugar 3 Io $\mathrm{mg}$./100 ml.), and considerable increases in the level of blood catecholamines as well as increased urinary excretion of catecholamines. The renal excretion of sodium was considerably lowered.
He was given deslanoside, hydrochlorothiazide, aminophylline, prenylamine, and, after 4 days, crystalline insulin ( 35 units daily) as well as methimazole ( $45 \mathrm{mg}$. daily) were added. His condition improved. The signs of cardiac failure disappeared completely after about 3 weeks, and he was discharged home after 6 weeks of treatment.

Case 5 A man of 4I was healthy until admitted to hospital with overwhelming coronary chest pain which appeared suddenly, followed by pulmonary oedema and ventricular fibrillation. After these had been overcome, symptoms of cardiac failure were seen during the next few days (cyanosis, lung congestion, gallop rhythm) as well as rhythm disorders (multifocal ventricular ectopic beats). Hormonal determinations indicated an outstanding increase in thyroid activity and particularly high adrenergic reaction in the first few days. At the same time a very low level of immunoradioactive insulin was found (blood sugar $296 \mathrm{mg}$./100 ml.). The urinary sodium excretion was considerably lowered.

On the fourth day besides treatment with acetyldigitoxin, dipyridamole, and aminophylline, crystalline insulin (6 units twice daily) was administered. The condition of the patient improved gradually. The raised hormone levels returned to normal. Urinary excretion of 17-OHCS decreased below normal levels in the third to fourth week and the disposition to the postural hypotension occurred, but disappeared later on.

\section{Patients suffering from myocardial infarc-} tion with a mild clinical course (Table 2 )

Case 6 A man of 47 , in hospital because of a recent myocardial infarction, had coronary chest pain for the first 3 days. His further course was favourable, and he was discharged after 5 weeks of treatment. Hormonal determinations did not show any considerable deviations from the normal apart from some transient increases in blood catecholamines.

Case 7 A man of 41 , who had had a myocardial infarction 5 years previously, was in hospital because of a recent myocardial infarction, preceded by a period of coronary chest pains lasting for about a fortnight. After 3 days the pain ceased, and he was discharged after 5 weeks of treatment. A slight and transient increase in PBI was noted during the first few days after the infarction as well as increased levels of catecholamines in the blood (the urinary excretion of catecholamines was not increased) and also the decreased excretion of sodium. These alterations disappeared at the end of the first week of the disease.

Case 8 A man of 48 had a myocardial infarction after several days of coronary insufficiency. For the first 4 days some single ventricular extrasystoles persisted. The further course of the disease was uncomplicated, and he was discharged after 6 weeks of treatment. No appreciable deviations from the normal were found in hormones. 
TABLE 2 Hormonal activity in patients with slight myocardial infarction

\begin{tabular}{|c|c|c|c|c|c|c|c|c|c|c|c|c|c|}
\hline & & \multicolumn{11}{|c|}{ Investigations } & \multirow{3}{*}{ Clinical symptoms } \\
\hline & & \multicolumn{5}{|l|}{ Blood } & \multicolumn{6}{|l|}{ Urine } & \\
\hline & & $\begin{array}{l}\text { Protein- } \\
\text { bound } \\
\text { iodine }\end{array}$ & $\begin{array}{l}\text { Hamol- } \\
\text { sky } \\
\text { test }\end{array}$ & $\begin{array}{l}\text { Immuno- } \\
\text { radio- } \\
\text { active } \\
\text { insulin }\end{array}$ & $\begin{array}{l}\text { Adrena- } \\
\text { line }\end{array}$ & $\begin{array}{l}\text { Nor- } \\
\text { adrena- } \\
\text { line }\end{array}$ & $\begin{array}{l}\text { Adrena- } \\
\text { line }\end{array}$ & $\begin{array}{l}\text { Nor- } \\
\text { adrena- } \\
\text { line }\end{array}$ & $\begin{array}{l}\text { Vanillyl } \\
\text { mandelic } \\
\text { acid }\end{array}$ & $\begin{array}{l}17- \\
\text { OHCS }\end{array}$ & $K+$ & $\mathrm{Na}+$ & \\
\hline \multirow[t]{6}{*}{$\begin{array}{c}\text { Case } 6 \\
\text { Days }\end{array}$} & 2 & $6 \cdot 9$ & - & - & $2 \cdot I$ & $9 \cdot 4$ & $11 \cdot 4$ & $29 \cdot 7$ & $4 \cdot 3$ & 5.5 & $2 \cdot 7$ & 0.9 & $\begin{array}{c}\text { Cardiac pain, circulatory } \\
\text { insufficiency }\end{array}$ \\
\hline & 4 & $6 \cdot 3$ & 16.5 & $20 \cdot 0$ & $2 \cdot 8$ & 3.8 & $6 \cdot 1$ & $10 \cdot 0$ & 3.5 & - & $\mathrm{I} \cdot 6$ & 0.5 & $\begin{array}{l}\text { Cardiac pain, circulatory } \\
\text { insufficiency }\end{array}$ \\
\hline & 6 & 6.5 & $12 \cdot 0$ & - & $1 \cdot 5$ & $5 \cdot 0$ & $1 \cdot 7$ & 34.8 & $4 \cdot 6$ & 3. I & $\mathbf{1} \cdot \mathbf{3}$ & 0.4 & - \\
\hline & 2 & 5.4 & 16.5 & - & 0.5 & 8.4 & - & - & $6 \cdot 0$ & 3.9 & 0.7 & 0.8 & - \\
\hline & 3 & - & - & $24 \cdot 0$ & - & - & $8 \cdot I$ & 15.2 & $7 \cdot 9$ & - & $1 \cdot 4$ & $4 \cdot 1$ & - \\
\hline & 4 & $5 \cdot 3$ & $10 \cdot 0$ & - & 0.8 & $10 \cdot 7$ & $9 \cdot 0$ & $25 \cdot 8$ & $8 \cdot 3$ & 5.5 & $r \cdot 3$ & $2 \cdot 9$ & - \\
\hline \multirow{6}{*}{$\begin{array}{c}\text { Case } 7 \\
\text { Days }\end{array}$} & & & & & & & & & & & & & \\
\hline & 2 & $7 \cdot 4$ & - & $20 \cdot 0$ & $2 \cdot 2$ & 13.7 & $I \cdot 4$ & $29 \cdot 6$ & 6.8 & $5 \cdot 1$ & $1 \cdot 8$ & $x \cdot 0$ & - \\
\hline & 4 & $8 \cdot 0$ & $17 \cdot 0$ & - & $6 \cdot 2$ & $19 \cdot 7$ & $1 \cdot 0$ & 14.5 & $5 \cdot 0$ & $2 \cdot 1$ & 0.2 & I.4 & - \\
\hline & 6 & 5.9 & $12 \cdot 5$ & - & $3 \cdot 2$ & $5 \cdot 1$ & $\mathbf{I} \cdot \mathbf{I}$ & $28 \cdot 0$ & $4 \cdot 6$ & 4.8 & $1 \cdot 5$ & $1 \cdot 0$ & - \\
\hline & 2 & 5.9 & $13 \cdot 0$ & $34 \cdot 0$ & $2 \cdot 3$ & $3 \cdot 8$ & $9 \cdot 8$ & $28 \cdot 2$ & 4.8 & - & 2.I & 47 & - \\
\hline & 3 & 二 & - & - & $1 \cdot 0$ & 5.5 & $7 \cdot 7$ & $16 \cdot 1$ & $4 \cdot 1$ & $4 \cdot 0$ & $2 \cdot 3$ & $3 \cdot 3$ & - \\
\hline \multirow{3}{*}{$\begin{array}{c}\text { Case } 8 \\
\text { Days }\end{array}$} & & & & & & & & & & & & & \\
\hline & 2 & $5 \cdot 1$ & $16 \cdot 0$ & $26 \cdot 0$ & $\mathbf{I} \cdot \mathbf{I}$ & $4 \cdot 2$ & $I \cdot 8$ & 13.4 & 3.5 & $5 \cdot I$ & $I \cdot 0$ & 0.4 & $\begin{array}{l}\text { Cardiac pain, and } \\
\text { rhythm disturbances }\end{array}$ \\
\hline & 4 & $5 \cdot 0$ & 18.5 & - & 0.8 & $2 \cdot 8$ & 4.7 & 10.5 & $13 \cdot 2$ & 5.4 & $2 \cdot 0$ & 2.5 & $\begin{array}{l}\text { Cardiac pain, and } \\
\text { rhythm disturbances }\end{array}$ \\
\hline \multirow{3}{*}{ Weeks } & 6 & 6.8 & 13.5 & - & $\mathbf{I} \cdot \mathbf{0}$ & 3.7 & $I \cdot I$ & $58 \cdot 9$ & $5 \cdot 6$ & $4 \cdot 2$ & 2.8 & 2.4 & - \\
\hline & 2 & 5.2 & 16.5 & $28 \cdot 0$ & 0.7 & $3 \cdot 3$ & 0.7 & $67 \cdot 0$ & 5.7 & $5 \cdot 1$ & $\mathrm{I} \cdot 7$ & $4 \cdot 3$ & - \\
\hline & 3 & 5.4 & 12.5 & - & 0.4 & $3 \cdot 0$ & $3 \cdot 6$ & $32 \cdot 6$ & $14 \cdot 1$ & $5 \cdot 1$ & - & - & - \\
\hline \multirow{6}{*}{$\begin{array}{l}\text { Case } 9 \\
\text { Days }\end{array}$} & & & & & & & & & & & & & \\
\hline & $\begin{array}{l}2 \\
4\end{array}$ & $\begin{array}{l}5.0 \\
5 \cdot 0\end{array}$ & $\begin{array}{l}15 \cdot 0 \\
13 \cdot 0\end{array}$ & $28 \cdot 0$ & $\frac{3 \cdot I}{-}$ & 3.5 & $\underline{4 \cdot I}$ & $\underline{34} \cdot 3$ & 143 & $=$ & $\underline{I} \cdot \mathbf{I}$ & $3 \cdot 7$ & Cardiac pain \\
\hline & 6 & $6 \cdot 5$ & 17.5 & - & $1 \cdot 0$ & 13.5 & $1 \cdot 5$ & $17 \cdot 2$ & $7 \cdot 1$ & - & $\mathbf{I} \cdot \mathbf{I}$ & $\bar{I} \cdot 2$ & - \\
\hline & 2 & 3.4 & $10 \cdot 0$ & - & 0.8 & 8.4 & 5.6 & $39 \cdot 4$ & $11 \cdot 4$ & $3 \cdot 3$ & $I \cdot 4$ & $2 \cdot 8$ & - \\
\hline & 3 & $4 \cdot 3$ & $13 \cdot 0$ & - & $1 \cdot 3$ & 4.8 & $3 \cdot 0$ & $30 \cdot 5$ & $4 \cdot I$ & - & $\mathrm{I} \cdot 5$ & 3.0 & - \\
\hline & 4 & - & $15 \cdot 0$ & $32 \cdot 0$ & 0.7 & $\mathbf{I} \cdot \mathbf{3}$ & $7 \cdot 2$ & $68 \cdot 7$ & $8 \cdot 8$ & 3.4 & $\mathbf{I} \cdot \mathbf{I}$ & $2 \cdot 6$ & - \\
\hline \multirow{7}{*}{$\begin{array}{c}\text { Case ro } \\
\text { Days }\end{array}$} & & & & & & & & & & & & & \\
\hline & 2 & $4 \cdot 4$ & $14 \cdot 0$ & - & 0.6 & $2 \cdot 0$ & 6.5 & 29.5 & $3 \cdot 8$ & $3 \cdot 4$ & $I \cdot 6$ & $3 \cdot 6$ & Cardiac pain, circulatory \\
\hline & 4 & - & - & - & 0.8 & $2 \cdot 0$ & $2 \cdot 9$ & $34 \cdot 8$ & 3.4 & - & $\mathbf{I} \cdot \mathbf{3}$ & I.O & - \\
\hline & 6 & 5.3 & $16 \cdot 0$ & - & 0.8 & $4 \cdot 6$ & $10 \cdot 6$ & 24.5 & $5 \cdot 6$ & $3 \cdot 7$ & $2 \cdot 6$ & $2 \cdot 8$ & - \\
\hline & 2 & 4.0 & $\bar{\pi}$ & - & 0.6 & 3.9 & $5 \cdot 6$ & $31 \cdot 6$ & $8 \cdot 1$ & 4.2 & 3.7 & $3 \cdot 6$ & - \\
\hline & 3 & $5 \cdot 8$ & $16 \cdot 0$ & - & 0.8 & 4.8 & - & $21 \cdot 5$ & 3.8 & $3 \cdot 2$ & $I \cdot 9$ & 2.9 & - \\
\hline & 4 & 一 & 一 & - & 0.3 & $\mathbf{I} \cdot \mathbf{0}$ & $4 \cdot 7$ & $57 \cdot 7$ & $4 \cdot 8$ & $6 \cdot 0$ & $r \cdot 3$ & $3 \cdot 3$ & - \\
\hline $\begin{array}{l}\text { Normal } \\
\text { ranges }\end{array}$ & & $\begin{array}{c}5 \cdot 3 \pm 1 \cdot 7 \\
\mu \mathrm{g} . / 100 \\
\mathrm{ml} .\end{array}$ & $\begin{array}{c}15 \cdot 0 \pm 2 \cdot 5 \\
\%\end{array}$ & $\begin{array}{c}20-30 \\
\mu U^{20}\end{array}$ & $\begin{array}{c}0.6 \pm 0.5 \\
\mu \mathrm{g} .11 .\end{array}$ & $\begin{array}{c}2 \cdot 5 \pm 1 \cdot 1 \\
\mu \mathrm{g} . / 1\end{array}$ & $\begin{array}{c}3.5 \pm \mathrm{r} \cdot 3 \\
\mu \mathrm{g} \cdot / 24 \\
\mathrm{hr} .\end{array}$ & $\begin{array}{c}26 \cdot 9 \pm 7 \cdot 7 \\
\mu \mathrm{g} . / 24 \\
\mathrm{hr} .\end{array}$ & $\begin{array}{c}4 \cdot 6 \pm 1 \cdot 2 \\
\mathrm{mg} \cdot / 24 \\
\mathrm{hr} .\end{array}$ & $\begin{array}{c}5 \cdot 0 \pm 1 \cdot 5 \\
\text { mg./24 } \\
\text { hr. }\end{array}$ & $\begin{array}{c}2 \cdot 0-3 \cdot 0 \\
\mathrm{~g} \cdot / 24 \\
\mathrm{hr} .\end{array}$ & $\begin{array}{l}3.0-5 \cdot 0 \\
\text { g. } / 24 \mathrm{hr} .\end{array}$ & \\
\hline
\end{tabular}

Case 9 A man of 65 had a myocardial infarction after 3 days of coronary chest pains (for the first time in his life). On the next day the chest pain disappeared. His further course was without incident, and he was discharged after 5 weeks of treatment. The results of the hormonal determinations were within normal limits. A slight decrease in the urinary excretion of $17-\mathrm{OHCS}$ was noted, the value being close to the values usually found in hypoadrenia.

Case ro A man of 55 was admitted to hospital because of a recent myocardial infarction, with subsequent cardiac rhythm disturbances (ventricular ectopic beats). After 2 days all the clinical disorders ceased, and he was discharged after 6 weeks.

\section{Discussion}

As the present data indicate, the endocrinological tests performed in patients suffering from recent myocardial infarction yield values that differ considerably from normal ones. In the cases with an unfavourable clinical course, the deviations from normal values are much greater, persist longer, and are usually of a polyglandular nature.

As is known, the catecholamines play an essential role in these disorders. The serious effect of pathologically increased blood catecholamines on the energetic patterns of the heart muscle (such as 'oxygen wasting effect') has been discussed (Raab, 1963). It seems that 
these effects may account for the rhythm disturbances in the course of myocardial infarction. In the patients in this study in whom increased values of catecholamines have been found (Cases I, 3, 4, and 5), the rhythm disturbances were more frequent and serious. This confirms earlier experimental and clinical observations (Ceremużyński, Staszewska-Barczak, and Herbaczynska-Cedro, 1969; Januszewicz et al., 1968; Staszewska-Barczak and Ceremużyński, I968; Valori et al., 1967). Arrhythmia also appeared in those patients in whom an increased urinary excretion of epinephrine was found. The relation between the values of urinary excretion of noradrenaline and vanillyl mandelic acid, as well as the level of blood catecholamines and the rhythm disturbances, were not distinctly stated. However, it has been shown earlier that an increased concentration of catecholamines in the blood after myocardial infarction is not always accompanied by their increased urinary excretion, which probably results from transient increases in the secretion of catecholamines (Markiewicz, Ceremużyński, and Kuch, in preparation). The high values of catecholamines in the urine might thus reflect the increased secretion of adrenaline lasting for a long time, which renders its biological action more complete.

From the reports of Porte et al. (1966) as well as other authors, infusions of adrenaline at low doses induce a considerable decrease in the activity of immunoradioactive insulin, in spite of the hyperglycaemia found at the same time. This phenomenon has been found in many experiments performed in animals as well as in men who were volunteers (Altszuler et al., 1967; Porte et al., 1966).

The hormonal tests reported here, performed immediately after myocardial infarction had occurred, showed that the phenomenon mentioned above occurred under the conditions of the natural disease. In Cases $1,2,3,4$, and 5, the high excretion of epinephrine was accompanied by the fall in immunoradioactive insulin in spite of hyperglycaemia.

The general condition of these patients was very serious during the first days of the infarction; this probably may be closely related to hormonal disorders disturbing the cellular metabolism, in the sense of the rapid exhaustion of the cellular energetic reserves with a decrease of their regeneration. Insulin was administered in Cases $I, 3,4$, and 5 in order to prevent this unfavourable influence on the clinical course of the disease.

Recently we have shown that at the beginning of a myocardial infarction the form of the glycaemic and immunoradioactive insulin curves (after glucose loading) corresponded to that found in latent diabetes. In some patients these values became spontaneously normal in the third or fourth week (Eawecki, Kuch, and Ceremużyński, in press).

In our other study (Tatoní, Ceremużyński, and Wiśniewska, 1969) when the insulin-like activity of serum (insulin-like activity, BullMerill biological method) was serially determined during the acute phase of myocardial infarction, a transient increase in insulin-like activity was observed during the first and second week of the disease. It was accompanied by a decrease in glucose assimilation coefficient (K). Immunoradioactive insulin and insulin-like activity behaved in opposite ways. This suggests that carbohydrate metabolism disturbances in patients with recent myocardial infarction could also be related to some unknown causes besides the inhibition of insulin secretion.

In myocardial infarction with an unfavourable course, lowered $p \mathrm{H}$ values are found as a rule, especially in shock. Metabolic acidosis is found in 66 per cent of patients with myocardial infarction during the first hours of the disease, according to Neaverson (1966). In such patients the prognosis is very poor. As is known, the secretion of catecholamines is particularly high under such conditions. It is possible that the mechanism by which acidosis arises in infarction of poor risk, and especially in shock, is also related to the inhibition of the secretion of endogenous insulin which could be proved by the observations in this report.

Many authors have found that the polarizing mixture containing, among others, insulin, yielded good effects in some patients with myocardial infarction. It is possible that in cases in which beneficial effects have been obtained, the severe clinical manifestations were mainly related to the deficiency of endogenous insulin, its secretion being inhibited by the vivid adrenergic response.

As proved by these investigations, distinct insulin deficiency is not a constant finding in myocardial infarction. Excessive secretion of epinephrine does not always occur in myocardial infarction either. It might be thought that treatment with the polarizing mixture would not give any essential benefits in such patients, which could account for different opinions on this method of treatment expressed in the literature.

Higher indexes of thyroid activity (PBI and Hamolsky-test) were found in some patients (Cases 3, 4, and 5) in whom increased secretion of catecholamines appeared after 
myocardial infarction. Biological synergy of catecholamines with thyroid hormones is well known. Both these groups of hormones enhance reciprocally their action on tissues, thus accelerating the catabolic processes that result in the depletion of the high energy bonds in the heart muscle.

In one patient (Case 3) in a poor clinical state, increased thyroid activity was found and hypoadrenia was shown simultaneously. As is clear from published reports, this is particularly dangerous for the heart. Many investigators have found that the activities of some enzymes in the heart (peroxidase, transferase, dehydrogenase) decrease distinctly when there is a deficiency of glycocorticoids (Davis, 1963; Hennes, 1962). Barta and Pavlovic̀va (I965) and Lefer, Verrier, and Carson (1968) have shown experimentally that the indexes of heart work in animals deteriorated considerably after adrenectomy, and that metabolic disturbances and circulatory failure soon appeared. It might be expected that the pathological manifestations mentioned above appear sooner when the thyroid action is increased, as well as leading to an additional charge for the heart.

The syndrome of thyroid hyperfunction, suprarenal insufficiency, and myocardial infarction, the so-called clinical triad, has been the subject of a report by Ceremużyński (1964). Until recently we have seen several such cases and think that this syndrome appears often though it is difficult to see clinically. The diagnosis can be made in these cases only with the use of routine hormonal monitoring in patients suffering from myocardial infarction.

The state of hypoadrenia even when it is not accompanied by hyperthyroidism is a very unfavourable phenomenon. In stress, glycocorticoid deficiency may even be dangerous, since these hormones are responsible for the adaptation processes. In one patient (Case 2) a few days before his death high and constantly rising urinary excretion of epinephrine was found. This might be considered the result of heavy stress (hypoxia, coronary chest pain). The excretion of 17-OHCS dropped much below the normal level at the same time. Glycocorticoids were administered on the last day before the patient's death, perhaps too late to exert any clinical effect.

The decrease in the excretion of the glycocorticoids, the so-called phase of hypoadrenia in myocardial infarction, was noted in the cases with poor risk in the second and third week after the infarction (Ceremużyński, I967a). The advantageous results of the treatment of such patients with substitute doses of cortisone acetate have been reported (Ceremużyński, 1967b).

The analysis of the results of the studies performed in the patients reported here showed hormonal disorders of the polyglandular type, leading probably to a disturbance of cellular metabolism during the stress induced by the formation of the myocardial infarction. It was possible to reach this conclusion by continuous 'hormonal monitoring' carried out by us in patients with recent myocardial infarction. The attempts to correct certain considerable hormonal disorders, especially in serious cases, yielded, it seems to us, positive results. These results encourage the introduction of the monitoring of some hormonal indexes as a rule in intensive cardiac care units, thus enabling them to be corrected.

\section{References}

Aleksandrow, D., Januszewicz, W., and Wocial, B. (1966). Urinary excretion of noradrenaline, adrenalin and vanillyl mandelic acid in myocardial infarction. (Polish.) Polskie Archiwum Medycyny Wewnętrznej, 36, 437.

Altszuler, N., Steele, R., Rathgeb, I., and De Bodo, R. C. (1967). Glucose metabolism and plasma insulin level during epinephrine infusion in the dog. American fournal of Physiology, 212, 677.

Arora, R. B. (1965). Role of aldosterone in myocardial infarction. Annals of the New York Academy of Sciences, 118, 537.

Barker, S. B., and Klitgaard, H. M. (1952). Metabolism of tissues excised from thyroxine-injected rats. American Fournal of Physiology, 170, 81.

Barta, E., and Pavlovicova, H. (I965). The role of the adrenals in maintaining the level of carbohydrate metabolism in the failing heart. Cor et Vasa, 7, 60.

Bertler, A., Carlsson, A., and Rosengren, E. (1958). A method for the fluorimetric determination of adrenaline and noradrenaline in tissues. Acta Physiologica Scandinavica, 44, 273.

Ceremuźyński, L. (1964). A clinical triad: adrenal insufficiency, hyperthyroidism, and myocardial infarct. (Polish.) Polskie Archiwum Medycyny Wewnẹtrznej, 34, 23 I.

- (1967a). Clinical course of myocardial infarction and the excretion of 17 ketogenic steroids and 17 ketosteroids in urine. Cor et Vasa, 9, II.

- (1967b). Indications for the use of glucocorticoids in the treatment of a recent myocardial infarct. (Polish.) Polskie Archiwum Medycyny Wewnętrznej, 38, 5 .

(1970). Catecholamines in blood and urine, thyroid and suprarenal gland activity, insulin (IRI) level in patients with myocardial infarction with good and serious clinical course. Annals of Medical Section of Polish Academy of Sciences. In the press. -, Staszewska-Barczak, J., and HerbaczynskaCedro, K. (1969). Cardiac rhythm disturbances and the release of catecholamines after acute coronary occlusion in dogs. Cardiovascular Research, 3, 190.

Davis, V. E. (1963). Effect of cortisone and thyroxine on aromatic amino acid decarboxylation. Endocrinology, 72, 33. 
Gazes, P. C., Richardson, J. A., and Woods, E. F. (I959). Plasma catechol amine concentrations in myocardial infarction and angina pectoris. Circulation, 19, 657.

Hamolsky, M. W., Stein, M., and Freedberg, A. S. (1957). The thyroid hormone-plasma protein complex in man. II. A new in vitro method for study of 'uptake' of labelled hormonal components by human erythrocytes. Fournal of Clinical Endocrinology and Metabolism, 17, 33.

Hennes, A. R. (1962). Abnormalities of acetate metabolism in adrenal insufficiency in man. American Fournal of Medicine, 32, 343.

Januszewicz, W., Sznajderman, M., Wocial, B., and Preibisz, J. (1968). Urinary excretion of free norepinephrine and free epinephrine in patients with acute myocardial infarction in relation to its clinical course. American Heart fournal, 76, 345.

Klein, A. J., and Palmer, L. A. (1963). Plasma cortisol in myocardial infarction. A correlation with shock and survival. American fournal of Cardiology, 11, 332.

Kuschke, H. J., and Schneider, K. W. (1960). Die Sympathiko-adrenale Reaktion beim Herzinfarkt. Zeitschrift für Kreislaufforschung, 49, $26 \mathrm{r}$.

Ławecki, J., Kuch, J., and Ceremużyrski, L. (In the press). Immuno-reactive insulin (IRI) after oral glucose loading in the first and fourth week after onset of myocardial infarction. Polskie Archiwum Medycyny Wewnętrznej.

Lefer, A. M., Verrier, R. L., and Carson, W. W. (1968). Cardiac performance in experimental adrenal insufficiency in cats. Circulation Research, 22, 817.

Logan, R. W., and Murdoch, W. R. (I966). Bloodlevels of hydrocortisone, transaminases, and cholesterol after myocardial infarction. Lancet, 2, 52I.

Markiewicz, L., Ceremużyński, L., and Kuch, J. (In preparation). Secretion and the excretion of catecholamines in myocardial infarction in men.
Melani, F., Ditschuneit, H., Bartelt, K. M., Friedrich, H., and Pfeiffer, E. F. (1965). Úber die radioimmunologische Bestimmung von Insulin im Blut. Klinische Wochenschrift, 43, 1000.

Neaverson, M. A. (1966). Metabolic acidosis in acute myocardial infarction. British Medical fournal, 2, 383.

Porte, D., Jr., Graber, A. L., Kuzuya, T., and Williams, R. H. (I966). The effect of epinephrine on immunoreactive insulin levels in man. Fournal of Clinical Investigation, 45, 228.

Raab, W. (1963). The nonvascular metabolic myocardial vulnerability factor in 'coronary heart disease'. American Heart Fournal, 66, 685.

Silber, R. H., and Porter, C. C. (1954). The determination of 17,21-dihydroxy-20-ketosteroids in urine and plasma. fournal of Biological Chemistry, 210, 923.

Staszewska-Barczak, J., and Ceremużyński, L. (1968). The continuous estimation of catecholamine release in the early stages of myocardial infarction in the dog. Clinical Science, 34, 531.

Tatoń, J., Ceremużyński, L., and Wiśniewska, A. (1969). Zaburzenia przemiany wȩglowodanowej w ostrym okresie zawału serca. Polskie Archiwum Medycyny Wewnętrznej, 43, 1579.

Woiwod, A. J., and Knight, R. (196I). The determination of 3-methyoxy 4-hydroxy mandelic acid in urine. Fournal of Clinical Pathology, 14, 502.

Valori, C., Thomas, M., and Shillingford, J. (1967). Free noradrenaline and adrenaline excretion in relation to clinical syndromes following myocardial infarction. American fournal of Cardiology, 20, 605.

Wolff, H. P., Koczorek, K. R., Buchborn, E., and Köhler, M. (I956). UUber die Aldosteronaktivität und Natriumretention bei Herzkranken und ihre Pathophysiologische Bedeutung. Klinische Wochenschrift, 34, I I05. 